\title{
Evaluation of three-dimensional acromiohumeral distance in the standing position and comparison with its conventional measuring methods
}

Yuki Yoshida ${ }^{1}$, Noboru Matsumura ${ }^{1 *} \mathbb{D}$, Yoshitake Yamada ${ }^{2 *}$, Minoru Yamada ${ }^{2}$, Yoichi Yokoyama $^{2}$, Morio Matsumoto ${ }^{1}$, Masaya Nakamura', Takeo Nagura ${ }^{1}$ and Masahiro Jinzaki ${ }^{2}$

\begin{abstract}
Background: Narrowing of the acromiohumeral distance (AHD) implies a rotator cuff tear. However, conventional AHD measurements using two-dimensional (2D) imaging or with the patient in the supine position might differ from that while standing during daily activity. This study aimed to evaluate the three-dimensional (3D) actual distance between the acromion and humeral head in the standing position and compare the AHD values with those obtained using conventional measuring methods.

Methods: Computed tomography (CT) images of 166 shoulders from 83 healthy volunteers (31 male and 52 female; mean age $40.1 \pm 5.8$ years; age range, 30-49 years) were prospectively acquired in the supine and standing positions using conventional and upright CT scanners, respectively. The minimum distance between the acromion and humeral head on the 3D surface models was considered as the 3D AHD. We measured the 2D AHD on anteroposterior digitally reconstructed radiographs. The AHD values were compared between the supine and standing positions and between the 2D and 3D measurements.

Results: The mean values of 2D AHD were $8.8 \pm 1.3 \mathrm{~mm}$ (range, $5.9-15.4 \mathrm{~mm}$ ) in the standing position and $8.1 \pm$ $1.2 \mathrm{~mm}$ (range, $5.3-14.3 \mathrm{~mm}$ ) in the supine position. The mean values of 3D AHD were $7.3 \pm 1.4 \mathrm{~mm}$ (range, 4.7$14.0 \mathrm{~mm}$ ) in the standing position and $6.6 \pm 1.2 \mathrm{~mm}$ (range, 4.4-13.7 mm) in the supine position. The values of 3D AHD were significantly lower than those of $2 \mathrm{D}$ AHDs in both the standing and supine positions $(P<0.001)$. The values of 2D and 3D AHDs were significantly lower in the supine position than in the standing position $(P<0.001)$.

Conclusions: This study evaluated the 3D AHD of normal shoulders in the standing position using an upright CT scanner. The present results indicated that assessments in the supine position can underestimate the value of the AHD compared with those made in the standing position and that assessments using 2D analysis can overestimate the value.
\end{abstract}

Keywords: Normal shoulder, Acromiohumeral distance, Acromiohumeral interval, Upright computed tomography, Position, Digitally reconstructed radiographs

\footnotetext{
*Correspondence: noboru18@gmail.com; yamada@rad.med.keio.ac.jp

'Department of Orthopedic Surgery, Keio University School of Medicine, 35

Shinanomachi, Shinjuku-ku, Tokyo 160-8582, Japan

${ }^{2}$ Department of Radiology, Keio University School of Medicine, 35

Shinanomachi, Shinjuku-ku, Tokyo 160-8582, Japan
}

(C) The Author(s). 2020 Open Access This article is licensed under a Creative Commons Attribution 4.0 International License, which permits use, sharing, adaptation, distribution and reproduction in any medium or format, as long as you give appropriate credit to the original author(s) and the source, provide a link to the Creative Commons licence, and indicate if changes were made. The images or other third party material in this article are included in the article's Creative Commons licence, unless indicated otherwise in a credit line to the material. If material is not included in the article's Creative Commons licence and your intended use is not permitted by statutory regulation or exceeds the permitted use, you will need to obtain permission directly from the copyright holder. To view a copy of this licence, visit http://creativecommons.org/licenses/by/4.0/ The Creative Commons Public Domain Dedication waiver (http://creativecommons.org/publicdomain/zero/1.0/) applies to the data made available in this article, unless otherwise stated in a credit line to the data. 


\section{Introduction}

The acromiohumeral distance (AHD) is usually assessed on anteroposterior shoulder radiographs acquired in the standing position. Narrowing of this distance is widely considered to imply a rotator cuff tear [1-4]. However, the distances on radiographic two-dimensional (2D) measurements might differ from the actual distance between the humeral head and acromion [5]. Although a three-dimensional (3D) distance can be measured using computed tomography (CT), conventional CT scanners acquire images of the shoulder girdle only in the supine position [6, 7]. Moreover, it is possible that the AHD could change between the supine and standing positions owing to the effect of gravity and the rotation of the upper arm.

The minimum distance between the acromion and humeral head in the standing position has not been evaluated using a 3D approach. However, a novel upright CT scanner has been developed, which enables $3 \mathrm{D}$ whole-torso cross-sectional scanning in the standing position [8] and evaluation of the effect of gravity on the human body [9]. This study aimed to clarify the $3 \mathrm{D}$ minimum distance between the acromion and humeral head of normal shoulders in the standing position using an upright CT scanner and compare the AHD values with those obtained using
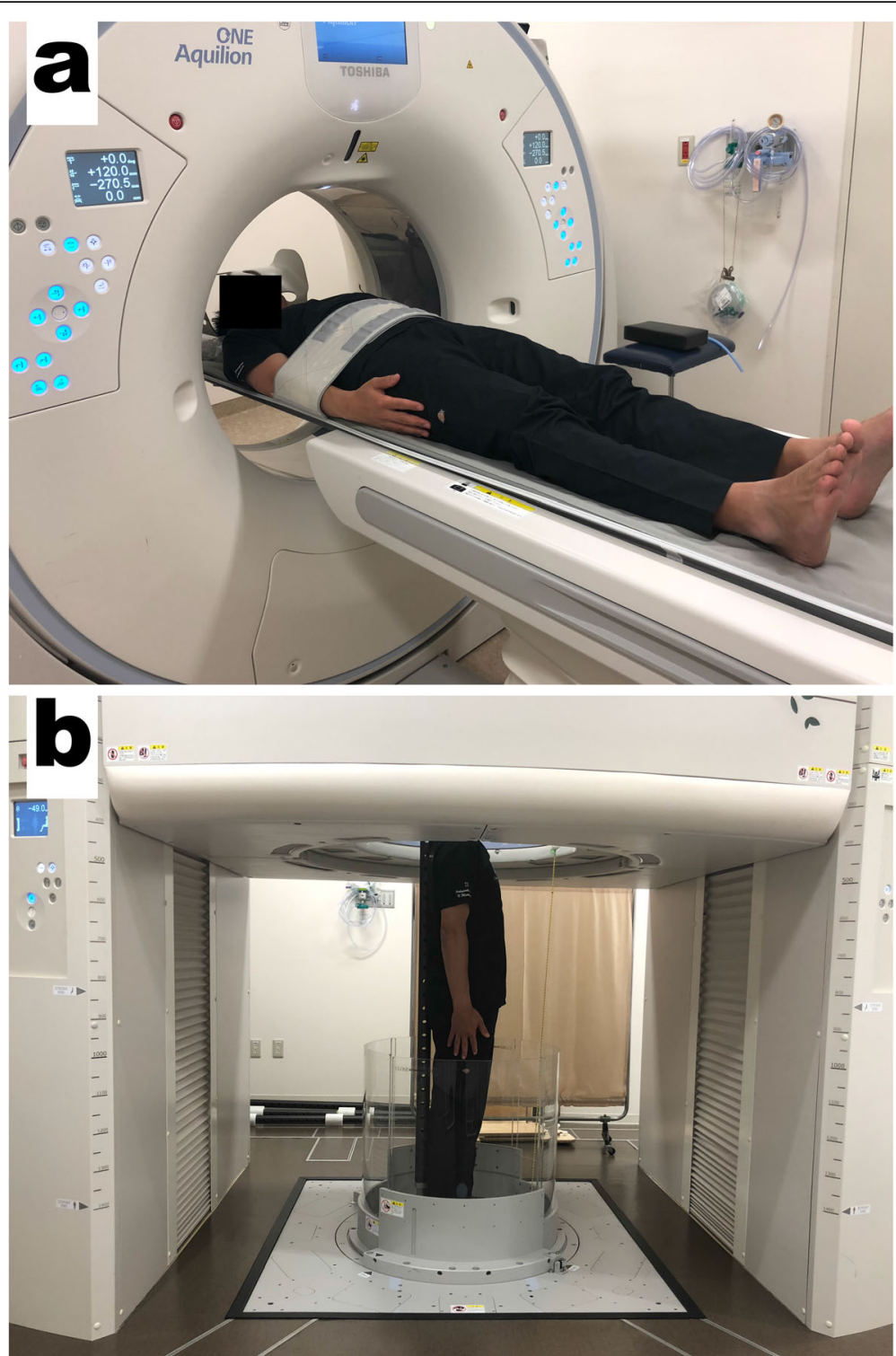

Fig. 1 320-row conventional and upright computed tomography (CT) scanners. CT images of the bilateral shoulders were obtained with the shoulders adducted and the arms held in a neutral position, both in the supine position using a conventional scanner (a) and in the standing position using an upright scanner (b) 
conventional methods. We hypothesized that 3D measurement of the actual distance between the acromion and humeral head in the standing position is feasible using CT scans and that its AHD values differ from those obtained using $2 \mathrm{D}$ analysis or in the supine position.

\section{Materials and methods}

\section{Participants}

This study was approved by the institutional review board, and written consent was obtained from all the participants (study protocol: \#20160384). The study was conducted between June 2017 and July 2018.

A total of 134 healthy male and female volunteers aged 30-89 years with no past illnesses or injuries to the shoulder girdle were recruited from a volunteer recruitment company. Of these, 50 participants aged over 50 years were excluded because of the increased risk of an asymptomatic rotator cuff tear or other degenerative changes in the shoulder girdle [10-12]. In addition, one volunteer aged 33 years was excluded because of defects in the shoulder observed on the CT image. Thus, 166
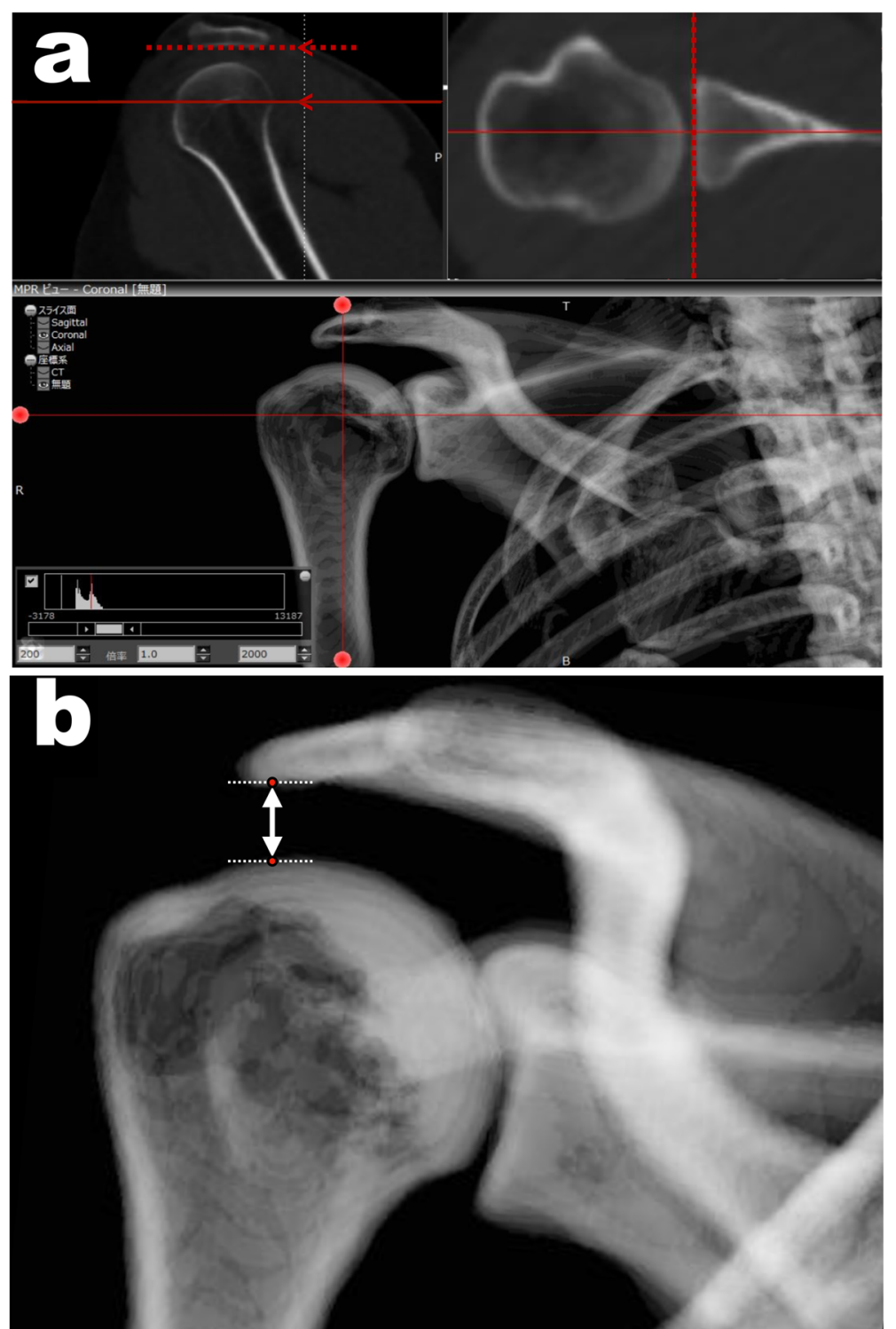

Fig. 2 The process of measuring the two-dimensional acromiohumeral distances (2D AHD). a Creating true anteroposterior shoulder digitally reconstructed radiograph (DRR) image from the $C T$ data using ZedView software. Scapula rotation was corrected to align parallel to the inferior aspect of the acromion in sagittal view and align parallel to the face of the glenoid in axial view. $\mathbf{b}$ The acromiohumeral distance (AHD) was measured using a two-dimensional (2D) approach on DRR images of the anteroposterior shoulder reconstructed from the CT scans. The 2D AHD was defined as the shortest distance from the dense cortical bone at the inferior aspect of the acromion to the most proximal articular cortex of the humeral head (white line with arrow) 
shoulders from 83 healthy volunteers ( 31 male and 52 female) were included in the analysis. The participants' mean ( \pm standard deviation) age, height, body weight, and body mass index (BMI) were $40.1 \pm 5.8$ years (range, $30-49$ years), $162.9 \pm 8.7 \mathrm{~cm}$ (range, $147.7-184.0 \mathrm{~cm}$ ), $59.6 \pm 12.5 \mathrm{~kg}$ (range, $37.8-106.8 \mathrm{~kg}$ ), and $22.4 \pm 3.7 \mathrm{~kg} /$ $\mathrm{m}^{2}$ (range, $15.7-33.7 \mathrm{~kg} / \mathrm{m}^{2}$ ), respectively.

\section{Image acquisition}

Imaging of the bilateral shoulders was acquired for each participant using a conventional 320-detector-row CT scanner (Aquilion ONE; Canon Medical Systems Corporation, Otawara, Japan) in the supine position and an upright CT scanner (prototype TSX-401R; Canon Medical Systems Corporation, Otawara, Japan) in the standing position on the same day (Fig. 1a, b). During acquisition, the shoulders were adducted and the arms held in neutral position. The CT data were accumulated in Digital Imaging and Communication in Medicine (DICOM) data format.

\section{Measurement of AHD}

AHD was measured in 2D from digitally reconstructed radiograph (DRR) images of the anteroposterior (AP) shoulder radiographs, reconstructed from the DICOM data using ZedView software (version 12.5.0; LEXI, Tokyo, Japan). Multiplanar reformatting was used to create true AP shoulder DRR images, and scapula rotation was corrected for each individual glenoid version to provide a true AP view aligned parallel to the face of the glenoid (Fig. 2a). The bone window width set as 2000 Hounsfield units (HU) and the window level as $200 \mathrm{HU}$. The AHD was measured from the dense cortical bone at the inferior aspect of the acromion to the most proximal articular cortex of the humeral head, with the shortest distance recorded as the 2D AHD (Fig. 2b) $[2,3,5,13]$.

Furthermore, 3D bone surface models of the acromion and humeral head, which were extracted from the DICOM data using AVIZO software (version 9.3.0; Maxnet, Tokyo, Japan), were used for measuring the $3 \mathrm{D}$ AHD. Bone part segmentation was performed to observe each slice of multiplanar reformatting carefully, with the bone window width set as $2000 \mathrm{HU}$ and the window level as $200 \mathrm{HU}$ (Fig. 3a). The bone surface model was generated with the smoothing level setting of 1.75 , and the scapula and the humerus surface models were exported as Standard Triangulated Language (STL) data (Fig. 3b). To evaluate the 3D distance between the acromion and the humeral head, the STL data for the glenoid and coracoid parts of the scapula surface models were removed using Meshlab software (version 1.3.3; ISTI, Pisa, Italy). The minimum distance of these two bone surface models was automatically computed as the Hausdorff distance using the Meshlab software [14-16].

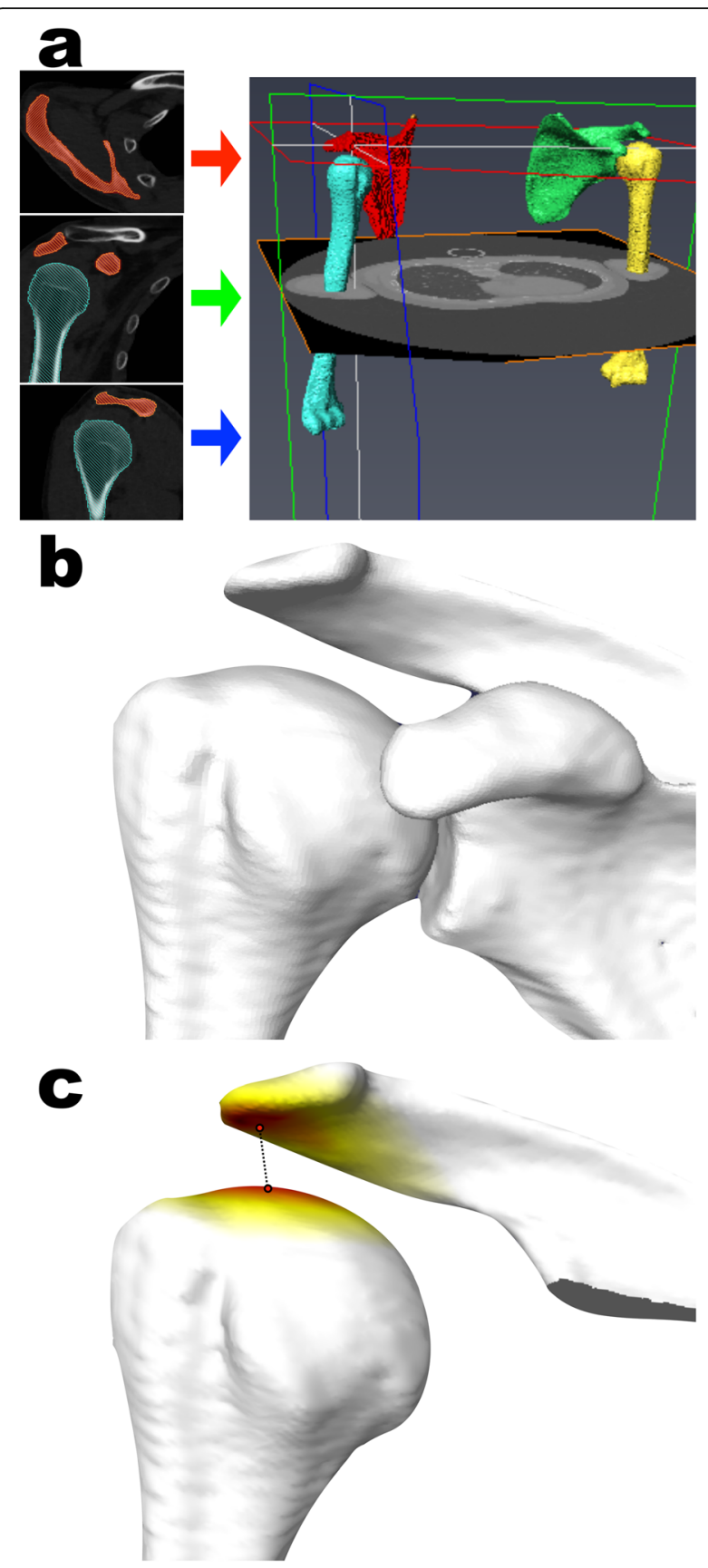

Fig. 3 The process of calculating the three-dimensional acromiohumeral distances (3D AHD). a Creating 3D surface models of the scapula and humerus from the $C T$ data to observe three views of multiplanar reformatting carefully using AVIZO software. $\mathbf{b}$ Bone surface model of the scapula and proximal humerus. c After removing the glenoid and coracoid parts of the surface model, the 3D AHD was automatically measured as the minimum distance between the acromion and humeral head on the software. The red areas indicate where the distance between the acromion and the humeral head is at a minimum

This distance indicates that any point in the acromion surface model can be reached at any point in the humeral head surface model by advancing at least the 
distance. The closest points between the acromion and humeral head were generated using the Hausdorff sampling filter, and the value of the minimum vertex quality was recorded as the 3D AHD (Fig. 3c).

\section{Statistical analysis}

SPSS Statistics 25.0.0.0 software (IBM Corp., Armonk, NY, USA) was used for the statistical analyses. The intra- and inter-observer reliabilities for the 2D and 3D AHD values were assessed by calculating intraclass correlation coefficient (ICC) based on 20 randomly selected shoulders. The measurements were made blind by two shoulder surgeons (ICC model 2,1) and repeated after a 3 -month interval by one shoulder surgeon (ICC model $1,1)$. After the reliabilities were determined to be acceptable, the 2D and 3D AHD values for all 166 shoulders were assessed by a single shoulder surgeon.

The AHD data did not present normal distribution using the Shapiro-Wilk test $(P<0.05)$, and nonparametric tests were performed. The differences in age, height, weight, BMI, and AHD values between the men and women were assessed using the Mann-Whitney $U$ test. The differences in the AHD values between the 2D and $3 \mathrm{D}$ measurements and between those measured in the supine and standing positions were evaluated using Wilcoxon signed-rank tests. Correlations in AHD values between the right and left shoulders were analyzed using Spearman's rank correlation analysis. The relationships between the AHD values and the participants' heights, weights, and BMI were also evaluated using Spearman's rank correlation analysis. The significance level was set at 0.05 for all the analyses.

\section{Results}

The intra- and inter-observer correlation coefficients for the 2D AHD measurements were 0.865 (95\% confidence interval $[\mathrm{CI}], 0.695-0.944)$ and 0.831 (95\% CI, $0.662-$ 0.930), respectively. Those for the 3D AHD measurements were 0.979 (95\% CI, 0.940-0.992) and 0.992 (95\% CI, 0.981-0.997), respectively. These results confirmed that the measurements of 2D and 3D AHD were highly reproducible.

The mean values for the 2D AHDs were significantly higher in the standing position than in the supine position, at $8.8 \pm 1.3 \mathrm{~mm}$ (range, $5.9-15.4 \mathrm{~mm}$ ) and $8.1 \pm$ $1.2 \mathrm{~mm}$ (range, $5.3-14.3 \mathrm{~mm}$ ), respectively; similarly, the mean values for the 3D AHDs were significantly higher in the standing position than in the supine position, at $7.3 \pm 1.4 \mathrm{~mm}$ (range, $4.7-14.0 \mathrm{~mm}$ ) and $6.6 \pm 1.2 \mathrm{~mm}$ (range, 4.4-13.7 mm), respectively (Fig. 4). The 3D AHD values were significantly lower than those for the $2 \mathrm{D}$ AHDs in both the standing and supine positions (both $P$ $<0.001$ ). The individual differences between the $2 \mathrm{D}$ and 3D AHD values obtained in the standing position ranged widely from -0.4 to $3.3 \mathrm{~mm}$ (Fig. 5a). The individual differences between the 3D AHD values obtained in the supine position and those obtained in the standing

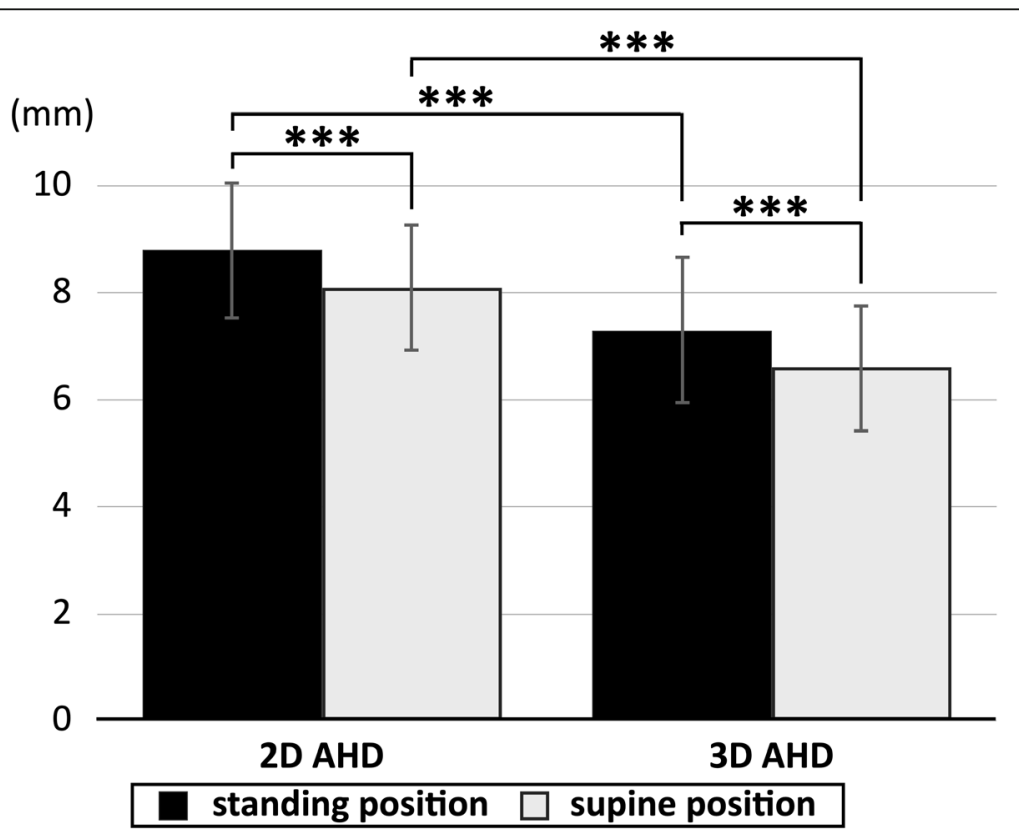

Fig. 4 Differences in acromiohumeral distances (AHD) between 2D and 3D measurements and between the supine and standing positions. The $3 \mathrm{D}$ AHD values were significantly lower than the $2 \mathrm{D}$ AHD values in both the standing and supine positions. The $2 \mathrm{D}$ and $3 \mathrm{D}$ AHD values were significantly lower in the supine position than in the standing position. ${ }^{* * *} P<0.001$ 
position showed even more variation, from -4.9 to 0.8 mm (Fig. 5b).

The mean values of both the $2 \mathrm{D}$ and the $3 \mathrm{D}$ AHD measurements were significantly lower for the women than for the men in both the standing and supine positions (Table 1).

Strong correlations were observed between the right and left shoulders for both the 2D and 3D AHDs, standing and supine (2D AHD: standing, $R=0.794, P<0.001$; supine, $R=0.780, P<0.001$; 3D AHD: standing, $R=0.711$, $P<0.001$; supine, $R=0.742, P<0.001$ ) (Fig. 6a,b). There was a weak correlation between the participants' height and the standing 3D AHD values, but not with any of the other AHD values (2D AHD: standing, $R=0.098, P=$
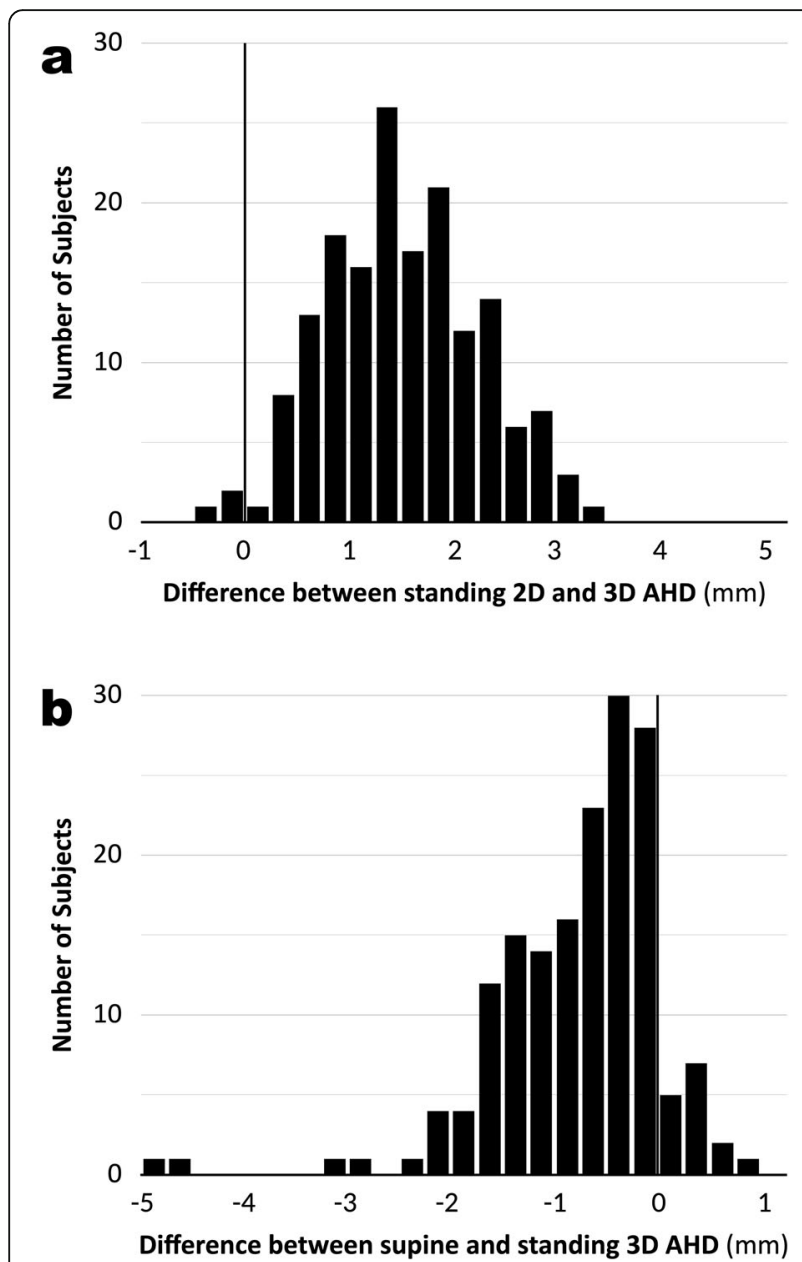

Fig. 5 Histogram of the individual differences in acromiohumeral distance (AHD). a Histogram of the individual differences in AHD in the standing position between the $2 \mathrm{D}$ and $3 \mathrm{D}$ measurements. Positive values indicate the $2 \mathrm{D}$ value is greater than the $3 \mathrm{D}$ value. $\mathbf{b}$ Histogram of the individual differences in 3D AHD between the supine and standing positions. Positive values indicate that the standing value is greater than the supine value. The differences varied widely
0.211; supine, $R=0.137, P=0.079$; 3D AHD: standing, $R$ $=0.161, P=0.038$; supine, $R=0.149, P=0.055)$. Weak correlations were observed between the ADH values and the participants' weight (2D AHD: standing, $R=0.299, P$ $<0.001$; supine, $R=0.386, P<0.001$; 3D AHD: standing, $R=0.319, P<0.001$; supine, $R=0.310, P<0.001)$ and BMI (2D AHD: standing, $R=0.303, P<0.001$; supine, $R=$ $0.386, P<0.001$; 3D AHD: standing, $R=0.284, P<0.001$; supine, $R=0.290, P<0.001$ ).

\section{Discussion}

This study evaluated the shortest distance between the acromion and humeral head in the standing position by using a newly developed upright CT scanner with a 3D approach $[8,9]$. Measuring 3D AHD while standing straight was feasible, and the mean value of the 3D AHD of healthy participants without previous injuries was $7.3 \mathrm{~mm}$, ranging from 4.7 to $14.0 \mathrm{~mm}$. The AHD was greater in men than in women. These results were compared with values of AHD evaluated using a $2 \mathrm{D}$ method in both the standing and supine positions and using a 3D method in the supine position. The 2D measurements were significantly higher than the 3D measurements, and the supine measurements were significantly lower than the standing measurements. Hence, the 2D analysis can overestimate the shortest distance between the acromion and the humeral head, and assessment in the supine position can underestimate the AHD compared with that in the standing position.

The normal value of AHD calculated using anteroposterior radiographs has been reported as $6-14 \mathrm{~mm}[1-4$, $17,18]$. The values of $2 \mathrm{D}$ AHD evaluated in the present study were consistent with these previous reports, but they were significantly greater than the values for $3 \mathrm{D}$ AHD, which are automatically computed assessment of the minimum distance between the 3D surfaces of the acromion and the humeral head. The individual differences between the 2D and 3D AHD values measured in the standing position varied widely by up to $3.3 \mathrm{~mm}$. According to the present findings, conventional 2D analysis cannot detect the actual shortest points of the acromion and humeral head because the inferior surfaces of these have a curved structure, which can be observed using 3D imaging [5].

The values of both 2D and 3D AHDs in the standing position were significantly higher than those in the supine position. In the standing position, gravity is likely to result in the humeral head moving downwards. The individual differences in 3D AHD between the standing and supine positions also varied widely, by up to 4.9 $\mathrm{mm}$, suggesting that the alignment changes in the glenohumeral joint between positions vary between individuals. In cases of rotator cuff tear, the patients often 
Table 1 Participant characteristics and two-dimensional (2D) and three-dimensional (3D) measurements of the acromiohumeral distance (AHD) made from images acquired in the standing and supine positions

\begin{tabular}{llllll}
\hline & & All, $\boldsymbol{N}=\mathbf{8 3}$ & Male, $\boldsymbol{n}=\mathbf{3 1}$ & Female, $\boldsymbol{n}=\mathbf{5 2}$ & $\boldsymbol{P}$ (sex difference) \\
\hline Characteristic & Age, years & $40.2 \pm 5.8$ & $39.4 \pm 4.9$ & $40.7 \pm 6.2$ & .154 \\
& Height, $\mathrm{cm}$ & $163.0 \pm 8.8$ & $171.5 \pm 6.0$ & $157.9 \pm 5.6$ & $<.001^{* * *}$ \\
& Weight, $\mathrm{kg}$ & $59.7 \pm 12.5$ & $69.7 \pm 11.8$ & $53.8 \pm 8.5$ & $<.001^{* * *}$ \\
& BMl, $\mathrm{kg} / \mathrm{m}^{2}$ & $22.4 \pm 3.7$ & $23.7 \pm 3.8$ & $21.6 \pm 3.5$ & $<.001^{* * *}$ \\
2D AHD, mm & Standing position & $8.8 \pm 1.3$ & $9.1 \pm 1.4$ & $8.7 \pm 1.3$ & $.034^{*}$ \\
& Supine position & $8.1 \pm 1.2$ & $8.5 \pm 1.3$ & $7.8 \pm 1.1$ & $.001^{* *}$ \\
3D AHD, mm & Standing position & $7.3 \pm 1.4$ & $7.7 \pm 1.5$ & $7.1 \pm 1.4$ & $.003^{* *}$ \\
& Supine position & $6.6 \pm 1.2$ & $7.0 \pm 1.4$ & $6.3 \pm 1.1$ & $<.001^{* * *}$ \\
\hline
\end{tabular}

The data are presented as mean \pm standard deviation. $B M I$ body mass index, $A H D$ acromiohumeral distance. ${ }^{*} P<.05,{ }^{* *} P<.01,{ }^{* * *} P<.001$

suffer from pain at night and sleep disturbance when lying in the supine position [19]; therefore, Railhac et al. [20] stated that measuring AHD in the supine position is useful in detecting a rotator cuff tear. Different positions can change the subacromial pressure [21, 22], and the narrowing of the AHD in the supine position might increase pain.

This was the first study to evaluate the AHD in the standing position using CT imaging. Saupe et al. [13] and Werner et al. [23] reported that 3D AHD measurements on magnetic resonance imaging acquired in the supine position were $2.8 \mathrm{~mm}$ and $0.6 \mathrm{~mm}$ lower, respectively, than 2D AHD measurements made on radiographs in the standing position. Similarly, Ongbumrungphan et al. [7] reported that 3D AHD measurements on $\mathrm{CT}$ imaging acquired in the supine position were $1.7 \mathrm{~mm}$ lower than 2D AHD radiograph measurements in the standing position. However, the insight into whether differences in human position or those between $2 \mathrm{D}$ and $3 \mathrm{D}$ analyses caused AHD variability still remains unclear. This study indicated that these differences may be caused by a combination of AHD overestimation by $2 \mathrm{D}$ analysis and AHD underestimation in the supine position. We believe that 3D AHD measured in the standing position, which reflects the actual distance between the acromion and the humeral head during daily activity, would be beneficial and helpful for clarifying the complex function of the shoulder in future studies.

Similar to the past reports [3, 24], the values of AHD in the present study varied greatly between individuals, and the AHD values were smaller in women than in men. The values of 3D AHD had a weak but significant correlation with the participants' height, weight, and BMI. This showed that the values of AHD differ with individual physical status, but that other factors including the shape of acromion and rotation of the scapula affect the values. The values of AHD were strongly correlated between sides, confirming that the AHD of the contralateral shoulder can act as a reference when assessing the AHD in cases of unilateral shoulder pathology.

The present study had several limitations. First, although the participants were healthy volunteers without any shoulder symptoms, we could not evaluate whether they had asymptomatic rotator cuff tears. The age of the volunteers was limited to $30-49$ years because of high correlation between the onset of rotator cuff tears and increasing age and because rotator cuff tears are clinically associated with lower AHD values. Yamaguchi et al. [12] reported that the mean age for individuals with no rotator cuff tear was 48.7 years, whereas for those with a unilateral tear it was 58.7 years, and for those with a bilateral tear it was 67.8 years. We excluded the volunteers over 50 years of age to ensure we avoided including shoulders with asymptomatic rotator cuff tear or other shoulder pathology. The comparisons between 2D and 3D measurements and between positions in the present study may differ from those that would be obtained for shoulders with rotator cuff tear; further studies are needed to investigate this. The measurement of 2D AHD might be another limitation. This was evaluated on DRR images reconstructed from CT data. DRR can be used to obtain a true anteroposterior view [25], and a past validation study [26] demonstrated that DRR can substitute for radiographs; however, the images obtained in this way may differ from the conventional radiographs used in previous 2D analyses.

\section{Conclusions}

This study evaluated the 3D AHD of normal shoulders in the standing position by using an upright CT scanner. The present results indicated that, compared with the measurement of AHD made in the standing position 


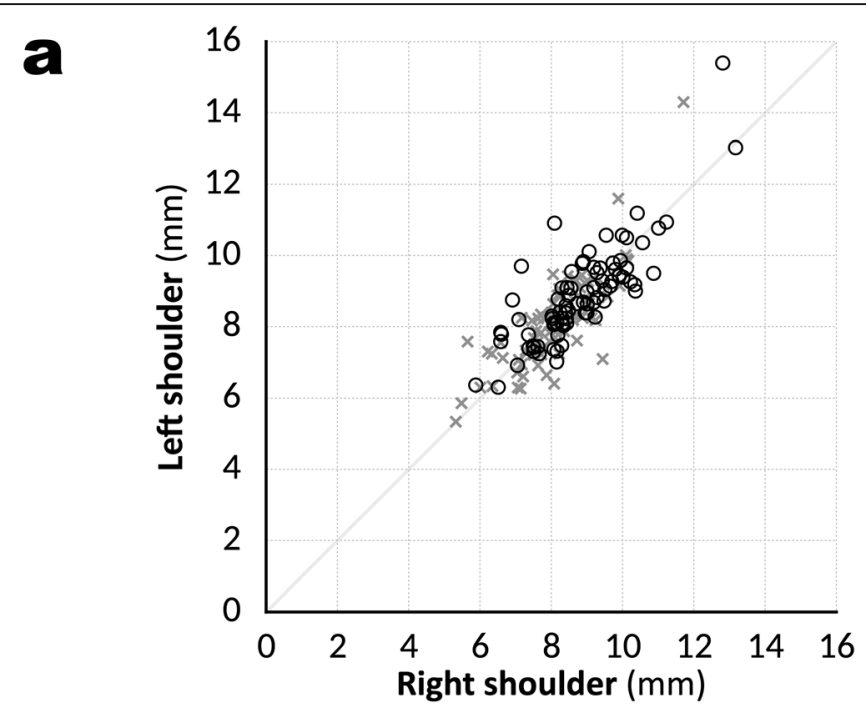

$O$ 2D AHD in standing position $\times$ 2D AHD in supine position

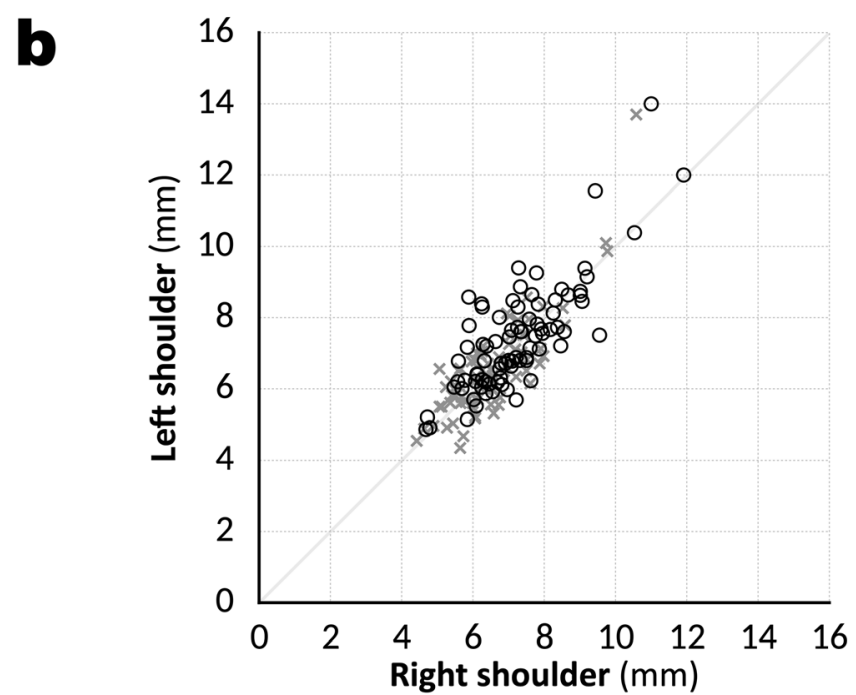

$O$ 3D AHD in standing position $\times$ 3D AHD in supine position

Fig. 6 Linear regression plots of acromiohumeral distance (AHD) values compared between the right and left shoulders. a Linear regression plots of acromiohumeral distance (AHD) values measured in two dimensions in the supine and standing positions, compared between the right and left shoulders. The values showed a strong correlation between the sides (standing: $R=0.794, P<0.001$; supine: $R=0.780, P<0.001$ ). $\mathbf{b}$ Linear regression plots for AHD values measured in three dimensions in the supine and standing positions, compared between the right and left shoulders. The values showed a strong correlation between the sides (standing: $R=0.711, P<0.001$; supine: $R=0.742, P<0.001$ )

with 3D analysis, measurements with $2 \mathrm{D}$ analysis can overestimate the value of AHD and assessments in the supine position can underestimate the value.

\section{Abbreviations}

AHD: Acromiohumeral distance; BMI: Body mass index; CT: Computed tomography; DICOM: Digital Imaging and Communication in Medicine; DRR: Digitally reconstructed radiograph; HU: Hounsfield units; ICC: Intraclass correlation coefficient; STL: Standard Triangulated Language; 2D: Twodimensional; 3D: Three-dimensional

\section{Acknowledgements \\ None}

Authors' contributions

Y. Yoshida: concept/design, data analysis, drafting of the manuscript, critical revision of the manuscript, and approval of the article. N. Matsumura: concept/design, validation, critical revision of the manuscript, and approval 
of the article. Y. Yamada: data collection, critical revision of the manuscript, and approval of the article. M. Yamada: data collection and approval of the article. Y. Yokoyama: data collection and approval of the article. M. Matsumoto: supervision and approval of the article. M. Nakamura: supervision and approval of the article. T. Nagura: supervision, critical revision of the manuscript, and approval of the article. M. Jinzaki: supervision and approval of the article.

\section{Funding}

This study was supported by Japan Society for the Promotion of Science (JSPS) KAKENHI (grant number JP17H04266, JP17K16482, and JP20K08056), Uehara Memorial Foundation, and Canon Medical Systems (Otawara, Japan).

\section{Availability of data and materials}

The datasets used and/or analyzed during the current study are available from the corresponding author on reasonable request.

\section{Ethics approval and consent to participate}

This study was approved by the Institutional Review Board of Keio University School of Medicine (reference study number 20160384), and written consent was obtained from all participants.

\section{Consent for publication}

Not applicable.

\section{Competing interests}

Masahiro Jinzaki has received a grant from Canon Medical Systems. However Canon Medical Systems was not involved in the design and conduct of the study; in the collection, analysis, and interpretation of the data; or in the preparation, review, and approval of the manuscript. The remaining authors have no conflicts of interest to declare.

\section{Received: 11 June 2020 Accepted: 31 August 2020}

Published online: 23 September 2020

\section{References}

1. Cotton RE, Rideout DF. Tears of the humeral rotator cuff; a radiological and pathological necropsy survey. J Bone Joint Surg Br. 1964;46:314-28.

2. Golding FC. The shoulder--the forgotten joint. Br J Radiol. 1962;35:149-58. https://doi.org/10.1259/0007-1285-35-411-149.

3. Petersson CJ, Redlund-Johnell I. The subacromial space in normal shoulder radiographs. Acta Orthop Scand. 1984;55:5758. https://doi.org/10.3109/ 17453678408992312

4. Weiner DS, Macnab I. Superior migration of the humeral head. A radiological aid in the diagnosis of tears of the rotator cuff. J Bone Joint Surg Br. 1970;52:524-7.

5. Bernhardt GA, Glehr M, Zacherl M, Wurnig C, Gruber G. Observer variability in the assessment of the acromiohumeral interval using anteroposterior shoulder radiographs. Eur J Orthop Surg Traumatol. 2013;23:185-90. https:// doi.org/10.1007/s00590-012-0942-y.

6. Li X, Xu W, Hu N, Liang X, Huang W. Jiang D, et al relationship between acromial morphological variation and subacromial impingement: a threedimensional analysis. PLoS One. 2017;12:e0176193. https://doi.org/10.1371/ journal.pone.0176193.

7. Ongbumrungphan W, Srikhum W, Chernchujit B. The effect of radiographic beam angle on acromiohumeral interval: 3D-CT analytic study. J Med Assoc Thail. 2015;98 Suppl 3;S61-5.

8. Jinzaki M, Yamada Y, Nagura T, Nakahara T, Yokoyama Y, Narita K, et al. Development of upright computed tomography with area detector for whole-body scans: phantom study, efficacy on workflow, effect of gravity on human body, and potential clinical impact. Investig Radiol. 2020;55:7383. https://doi.org/10.1097/rli.0000000000000603.

9. Yamada Y, Yamada M, Yokoyama Y, Tanabe A, Matsuoka S, Niijima Y, et al. Differences in lung and lobe volumes between supine and standing positions scanned with conventional and newly developed 320-detectorrow upright CT: intra-individual comparison. Respiration. 2020;99:598-605 https://doi.org/10.1159/000507265.

10. Gumina S, Carbone S, Campagna V, Candela V, Sacchetti FM, Giannicola G. The impact of aging on rotator cuff tear size. Musculoskelet Surg. 2013; 97(Suppl 1):69-72. https://doi.org/10.1007/s12306-013-0263-2.
11. Ogawa K, Yoshida A, Inokuchi W, Naniwa T. Acromial spur: relationship to aging and morphologic changes in the rotator cuff. J Shoulder Elb Surg. 2005;14:591-8. https://doi.org/10.1016/j.jse.2005.03.007.

12. Yamaguchi K, Ditsios K, Middleton WD, Hildebolt CF, Galatz LM, Teefey SA. The demographic and morphological features of rotator cuff disease. A comparison of asymptomatic and symptomatic shoulders. J Bone Joint Surg Am. 2006;88:1699-704. https://doi.org/10.2106/jbjs.E.00835.

13. Saupe N, Pfirrmann CW, Schmid MR, Jost B, Werner CM, Zanetti M. Association between rotator cuff abnormalities and reduced acromiohumeral distance. Am J Roentgenol. 2006;187:376-82. https://doi. org/10.2214/AJR.05.0435

14. Li J, Tang S, Zhang H, Li Z, Deng W, Zhao C, et al. Clustering of morphological fracture lines for identifying intertrochanteric fracture classification with Hausdorff distance-based K-means approach. Injury. 2019; 50:939-49. https://doi.org/10.1016/j.injury.2019.03.032.

15. Rino Neto J, Silva FP, Chilvarquer I, Paiva JB, Hernandez AM. Hausdorff distance evaluation of orthodontic accessories' streaking artifacts in 3D model superimposition. Braz Oral Res. 2012;26:450-6. https://doi.org/10. 1590/s1806-83242012000500012.

16. Simmons-Ehrhardt T, Falsetti C, Falsetti AB, Ehrhardt CJ. Open-source tools for dense facial tissue depth mapping of computed tomography models. Hum Biol. 2018:90:63-76.

17. Kotzen LM. Roentgen diagnosis of rotator cuff tear. Report of 48 surgically proven cases. Am J Roentgenol Radium Therapy, Nucl Med. 1971;112:50711. https://doi.org/10.2214/ajr.112.3.507.

18. Michener LA, McClure PW, Karduna AR. Anatomical and biomechanical mechanisms of subacromial impingement syndrome. Clin Biomech. 2003;18: 369-79.

19. Longo UG, Facchinetti G, Marchetti A, Candela V, Risi Ambrogioni L, et al. Sleep disturbance and rotator cuff tears: a systematic review. Medicina. 2019:55:453. https://doi.org/10.3390/medicina55080453.

20. Railhac JJ, Sans N, Rigal A, Chiavassa H, Galy-Fourcade D, Richardi G, et al. Strict anteroposterior radiography of the shoulder: value of the assessment of rotator cuff tears. J Radiol. 2001;82:979-85.

21. Holdaway LA, Hegmann KT, Thiese MS, Kapellusch J. Is sleep position associated with glenohumeral shoulder pain and rotator cuff tendinopathy: a cross-sectional study. BMC Musculoskelet Disord. 2018;19:408. https://doi. org/10.1186/s12891-018-2319-9.

22. Werner CM, Ossendorf C, Meyer DC, Blumenthal S, Gerber C. Subacromial pressures vary with simulated sleep positions. J Shoulder Elb Surg. 2010;19: 989-93. https://doi.org/10.1016/j.jse.2010.04.039.

23. Werner CM, Conrad SJ, Meyer DC, Keller A, Hodler J, Gerber C. Intermethod agreement and interobserver correlation of radiologic acromiohumeral distance measurements. J Shoulder Elb Surg. 2008;17:237-40. https://doi. org/10.1016/j.jse.2007.06.002.

24. Graichen $\mathrm{H}$, Bonel $\mathrm{H}$, Stammberger $\mathrm{T}$, Englmeier $\mathrm{KH}$, Reiser $\mathrm{M}$, Eckstein $\mathrm{F}$. Sex-specific differences of subacromial space width during abduction, with and without muscular activity, and correlation with anthropometric variables. J Shoulder Elb Surg. 2001;10:129-35. https://doi.org/10.1067/mse. 2001.112056

25. Suter T, Gerber Popp A, Zhang Y, Zhang C, Tashjian RZ, Henninger HB. The influence of radiographic viewing perspective and demographics on the critical shoulder angle. J Shoulder Elb Surg. 2015;24:e149-58. https://doi.org/ 10.1016/j.jse.2014.10.021.

26. Harris MD, Kapron AL, Peters $\mathrm{CL}$, Anderson AE. Correlations between the alpha angle and femoral head asphericity: implications and recommendations for the diagnosis of cam femoroacetabular impingement. Eur J Radiol. 2014;83:788-96. https://doi.org/10.1016/j.ejrad.2014.02.005.

\section{Publisher's Note}

Springer Nature remains neutral with regard to jurisdictional claims in published maps and institutional affiliations. 